\title{
Ensino de Machine Learning na Educação Básica: um Mapeamento Sistemático do Estado da Arte
}

\author{
Lívia S. Marques ${ }^{1}$, Christiane Gresse von Wangenheim ${ }^{1}$, Jean C. R. Hauck ${ }^{1}$ \\ ${ }^{1}$ Departamento de Informática e Estatística - Universidade Federal de Santa Catarina \\ (UFSC) \\ Campus Trindade - 88040-900 - Florianópolis - SC - Brasil \\ livia.marques@grad.ufsc.br, \{c.wangenheim, jean.hauck\}@ufsc.br
}

\begin{abstract}
As Machine Learning (ML) is present in various aspects of our lives, new challenges are presented to education in order to help students to understand its potential and limits. Thus, to obtain an overview of the state of the art on teaching ML concepts in $K$ 12, we carried out a systematic mapping. We identified 39 instructional units mostly focused on basic concepts and neural networks. Several instructional units also cover only partially the ML process, such as data management, or present certain processes only on an abstract level. Results demonstrate that teaching $M L$ in $K-12$ can increase understanding and interest as well as it contextualizes ML concepts in the lives of the students.
\end{abstract}

Resumo. Uma vez que a Machine Learning (ML) está presente em vários aspectos de nossas vidas, novos desafios são apresentados à educação em ajudar estudantes a entender os potenciais e limites dessa tecnologia. Para obter um panorama do estado da arte do ensino de ML, foi realizado um mapeamento sistemático. Foram identificadas 39 unidades instrucionais focadas em conceitos básicos e redes neurais. Muitas unidades abordam apenas de forma parcial o processo de ML, como gerenciamento de dados, ou apresentam alguns processos apenas de forma abstrata. Os resultados obtidos indicam que o ensino de ML na educação básica pode aumentar o entendimento e interesse, bem como contextualizar conceitos de ML na vida dos estudantes.

\section{Introdução}

Mesmo com a Inteligência Artificial (IA) se tornando parte do cotidiano e lhe trazendo diferentes e importantes impactos, poucas pessoas conhecem a tecnologia por trás dela [Evangelista et al. 2018]. Como consequência, têm-se a falsa impressão de que automação e IA são tópicos complexos e pouco acessíveis, de modo que as diversas possibilidades e benefícios que trazem fiquem em segundo plano. Portanto, é importante popularizar o entendimento básico das tecnologias de IA [Touretzky et al. 2019a]. Com isso, o ensino de computação ganha novas perspectivas e olhares, em busca de preparar os estudantes para serem mais do que consumidores das tecnologias, mas também criadores de soluções inovadoras e inteligentes [Touretzky et al. 2019b][Kandlhofer et al. 2016]. O ensino de IA na educação básica pode proporcionar um aumento no interesse desses estudantes em seguir carreiras nas áreas tecnológicas. O contato com essas tecnologias desde cedo oferece uma preparação mais sólida para essas possíveis futuras carreiras, que apresentam uma crescente demanda de profissionais com competências em IA [Forbes 2019]. Além disso, levar o ensino de IA para a educação básica representa uma democratização de acesso a esses conhecimentos, pois tende a incluir tradicionais minorias na área, como mulheres e pessoas em vulnerabilidade social e econômica. 
Muitas iniciativas atualmente abordam a programação de jogos e aplicativos, mas a educação básica ainda precisa abranger o ensino de conceitos básicos de IA. De acordo com AI4K12 [Touretzky et al. 2019c], cinco grandes ideias devem ser consideradas nesse ensino: percepção, representação e raciocínio, aprendizagem, interação natural e impactos sociais, incluindo na aprendizagem a Machine Learning (ML)[Wollowski et al. 2016][Touretzky et al. 2019a]. Machine Learning é a aplicação de Inteligência Artificial que confere aos sistemas a habilidade de automaticamente aprender e se desenvolver com base em experiências, sem serem explicitamente programados [Royal Society 2017]. Sendo assim, ML é uma abordagem encontrada em vários sistemas, desde reconhecimento de fala em assistentes inteligentes até veículos autônomos e sistemas de saúde, por exemplo.

Tradicionalmente, os conceitos básicos e técnicas de IA vêm sendo ensinados apenas no ensino superior [Torrey 2012][McGovern et al. 2011]. Porém, pode-se observar um recente movimento de inclusão desse tema também na educação básica ao redor do mundo. Novos cursos e ferramentas estão surgindo em diferentes países, e iniciativas como "AI for K-12 Initiative" [Touretzky et al. 2019c] estão começando a desenvolver diretrizes para o ensino de IA na educação básica.

Apesar de ser possível encontrar muitos desses projetos na internet, esses esforços ainda são bastante esparsos, sem que se tenha uma noção geral das unidades instrucionais específicas de IA existentes e suas características. Observa-se um foco maior das revisões de literatura no pensamento computacional [Lye \& Koh 2014][Grover \& Pea 2013][Heintz et al. 2016][Google 2016][Magno Jesus et al. 2019][Bordini et al. 2017], ou áreas de conhecimento relacionadas [Avila et al. 2017] [Ferreira et al. 2019], entre outras. Em relação ao ensino de IA/ML, as pesquisas existentes focam basicamente no ensino superior [Wollowski et al. 2016], de forma que não existem estudos sobre a aplicação do tema na educação básica. Outras revisões, como Tavares et al. (2020), focam no uso de ML para suportar qualquer tipo de ensino, mas não especificamente no ensino desse conhecimento.

Portanto, com o objetivo de responder a questão de se existem e quais são as unidades instrucionais atualmente disponíveis para o ensino de ML na educação básica, foi conduzido um estudo de mapeamento sistemático. A principal contribuição deste estudo é a síntese das características das unidades instrucionais (UI) para o ensino de ML do fundamental até o ensino médio, em relação ao seu conteúdo e contexto. Os resultados mostram que é possível e benéfico introduzir o ensino de ML na educação básica. Assim, esta visão geral apresentada pode ajudar professores a selecionar e/ou designers instrucionais a desenvolver unidades instrucionais. Espera-se que esta discussão possa promover ainda mais a inclusão do ensino de ML na educação básica.

\section{Definição e Execução do Mapeamento Sistemático}

Para levantar o estado da arte sobre se e como Machine Learning é abordada do ensino fundamental ao ensino médio, foi conduzido um mapeamento sistemático seguindo os procedimentos propostos por Petersen et al. (2008).

\subsection{Definição do protocolo de revisão}

A pergunta de pesquisa é: Quais unidades instrucionais existem para ensinar conceitos de Machine Learning no contexto do ensino fundamental até o ensino médio? Essa pergunta de pesquisa é refinada nas seguintes questões de análise:

AQ1. Quais UIs existem?

AQ2. Quais conceitos de Machine Learning são ensinados nessas UIs? 
AQ3. Quais são as características instrucionais dessas UIs?

Critérios de inclusão/exclusão. Foram consideradas quaisquer unidades instrucionais (curso, atividade, tutorial etc.) que tivessem foco no ensino de computação incluindo conceitos de ML do ensino fundamental até o ensino médio, publicadas em língua Inglesa entre 2010 e 2020. Unidades instrucionais que focam no ensino de ML no ensino superior e/ou que abordassem o ensino de computação sem aprofundar-se em conceitos de ML foram excluídas. Também foram excluídas publicações de blogs, vídeos ou ferramentas que não possuam uma unidade instrucional.

Critérios de qualidade. Foram considerados apenas artigos ou materiais com informações suficientes relacionadas a conceitos de ML, indicando, por exemplo, conteúdo de aulas, materiais de apoio etc.

Fontes dos dados. Foram examinados todos os materiais e artigos publicados em inglês e disponíveis nas mais importantes bibliotecas digitais acessíveis por meio do Portal Capes. O Google também foi incluído, por indexar um grande conjunto de dados de diferentes fontes [Haddaway et al. 2015], já que nessa área emergente muitas UIs não foram publicadas. Dado o foco de pesquisa do MIT Media Lab nessa área, publicações desse grupo também foram procuradas. Literatura secundária foi também incluída.

Definição da Search String. A search string foi composta de conceitos relacionados à questão de pesquisa, incluindo sinônimos. Dessas palavras chave, a search string foi sendo adaptada para cada fonte de dados apresentada na Tabela 1.

\section{Tabela 1. Search string para cada fonte}

\begin{tabular}{|c|c|}
\hline Source & Search string \\
\hline $\mathrm{ACM}$ & $\begin{array}{l}\text { [[Abstract: teach*] OR [Abstract: education] OR [Abstract: course] OR [Abstract: mooc] OR [Abstract: learn*]] AND } \\
\text { [[Abstract: "machine learning"] OR [Abstract: "data science"] OR [Abstract: "artificial intelligence"] OR [Abstract: "deep } \\
\text { learning"]] AND [[Abstract: "k-12"] OR [Abstract: school*] OR [Abstract: kids] OR [Abstract: children] OR [Abstract: teen*]] } \\
\text { AND [Publication Date: }(01 / 01 / 2010 \text { TO *)] }\end{array}$ \\
\hline IEEE & $\begin{array}{l}\text { ((("Abstract":teach*) OR ("Abstract":education) OR ("Abstract":course) OR ("Abstract":MOOC) OR ("Abstract":learn*)) AND } \\
\text { (("Abstract":“"machine learning") OR ("Abstract":“data science”) OR ("Abstract":“artificial intelligence”) OR ("Abstract":“deep } \\
\text { learning")) AND (("Abstract":“k-12") OR ("Abstract":school*) OR ("Abstract":kids) OR ("Abstract":children) OR } \\
(\text { ("Abstract":teen*)) ) } 2010 \text { - } 2020\end{array}$ \\
\hline Scopus & $\begin{array}{l}\text { TITLE-ABS-KEY ( ( teach* OR education OR course OR mooc OR learn*) AND ( "machine learning" OR "data } \\
\text { science" OR "artificial intelligence" OR "deep learning" ) AND ( "k-12" OR school* OR kids OR children OR teen*) } \\
\text { ) AND ( LIMIT-TO ( PUBYEAR, 2020) ) OR LIMIT-TO ( PUBYEAR, 2019) OR ... OR LIMIT-TO ( PUBYEAR } \\
\text {, } 2010 \text { ) ) AND ( LIMIT-TO ( SUBJAREA, "COMP" ) ) }\end{array}$ \\
\hline Google & "machine learning" teach "K-12" OR school \\
\hline MIT media lab ${ }^{1}$ & -- \\
\hline
\end{tabular}

\subsection{Execução da busca}

A pesquisa foi realizada em Junho de 2020 pelo primeiro autor e revisada pelos co-autores (Tabela 2). Algumas pesquisas retornaram muitos resultados mesmo depois da adaptação das search strings, devido ao fato de que artigos que descrevem como utilizar IA na educação, como no aprendizado de análise de dados, correspondem aos mesmos termos de busca.

Tabela 2. Número de artigos identificados por repositório e por fase de seleção

\begin{tabular}{|l|r|r|r|r|}
\hline \multicolumn{1}{|c|}{ Fonte } & $\begin{array}{c}\text { No. de resultados da } \\
\text { busca }\end{array}$ & $\begin{array}{c}\text { No. de resultados } \\
\text { analisados }\end{array}$ & \multicolumn{1}{c|}{$\begin{array}{c}\text { No. de resultados } \\
\text { potencialmente relevantes }\end{array}$} & $\begin{array}{c}\text { No. de resultados } \\
\text { relevantes }\end{array}$ \\
\hline ACM & 3,551 & 200 & 15 & 11 \\
\hline IEEE & 888 & 200 & 5 & 4 \\
\hline SCOPUS & 2,928 & 200 & 81 & 2 \\
\hline Google & $37,500,000$ & 500 & & 81 \\
\hline
\end{tabular}

\footnotetext{
${ }^{1}$ https://appinventor.mit.edu/explore/research, https://www.media.mit.edu/groups/lifelong-kindergarten/publications/
} 
IX Congresso Brasileiro de Informática na Educação (CBIE 2020)

Anais do XXXI Simpósio Brasileiro de Informática na Educação (SBIE 2020)

\begin{tabular}{|l|r|r|r|r|}
\hline MIT media lab & 141 & 148 & 6 & 4 \\
\hline
\end{tabular}

$\mathrm{Na}$ primeira fase de análise, títulos e resumos foram analisados, resultando em 114 artefatos potencialmente relevantes. No segundo estágio, os materiais foram lidos por inteiro, para assegurar sua relevância com respeito aos critérios de inclusão/exclusão. Documentos duplicados foram eliminados e aqueles que descrevem a mesma UI foram unificados. Como resultado, 39 UIs foram consideradas relevantes, como mostra a Tabela 3.

\subsection{Extração de dados}

Sistematicamente, dados foram extraídos dos artigos de forma a responder às questões de análise. A extração dos dados foi prejudicada em alguns momentos enquanto o material ia sendo encontrado, pois uma grande quantidade de UIs não foi publicada como artigos. Assim, informações foram extraídas com base no material instrucional disponível, inferindo-se características e objetivos de aprendizagem.

\section{Análise dos Dados}

\subsection{Quais unidades instrucionais existem?}

Um total de 39 unidades instrucionais abrangendo o ensino de ML do ensino fundamental até o ensino médio foram identificadas (Tabela 3). Algumas unidades instrucionais focam somente em ML, enquanto outras abordam conceitos de ML como parte de cursos mais completos de IA e/ou programação/engenharia de software.

\section{Tabela 3. Unidades Instrucionais de ensino de ML na educação básica}

\begin{tabular}{|c|c|c|}
\hline Referência & Nome da UI & Fonte \\
\hline (AI Family Challenge, 2019) & AI Family Challenge & https://www.curiositymachi-ne.org/about/ \\
\hline (ai4children, 2017) & AI 4 children & https://www.ai4children.org/ \\
\hline (AIinSchools, 2019) & AI in Schools & http://aiinschools.com/ \\
\hline (Apps for Good, 2019a) & $\begin{array}{l}\text { Apps for good: Machine Learning } \\
\text { in a day }\end{array}$ & https://www.appsforgood.org/courses/ml-in-a-day \\
\hline (Apps for Good, 2019b) & $\begin{array}{l}\text { Apps for good: Machine Learning } \\
\text { course }\end{array}$ & https://www.appsforgood.org/courses/machine-learning \\
\hline $\begin{array}{l}\text { (Burgsteiner et al., } \\
\text { 2016)(Burgsteiner, 2016) }\end{array}$ & $\begin{array}{l}\text { IRobot: Teaching the Basics of } \\
\text { Artificial Intelligence in High } \\
\text { Schools }\end{array}$ & $\begin{array}{l}\text { Burgsteiner, H. et al. (2016) Robot: Teaching the Basics of Artificial Intelligence in High } \\
\text { Schools. Proc. of the 6th Symposium on Educational Advances in Artificial Intelligence, } \\
\text { Phoenix, AZ, USA. Burgsteiner, H. (2016) Design and Evaluation of an introductory } \\
\text { artificial intelligence class in high schools. Thesis, TU Graz, Austria. }\end{array}$ \\
\hline (Code.org, 2019) & AI for Oceans & https://curriculum.code.org/hoc/plugged/9/ \\
\hline (Cognimates.me, 2019) & Cognimates & https://cognimate.me:2635/home \\
\hline (CS4FN, 2011) & $\begin{array}{l}\text { CS4FN - Computer Science for } \\
\text { Fun }\end{array}$ & $\begin{array}{l}\text { http://www.cs4fn.org/teachers/activities/braininabag/braininabag.pdf } \\
\text { http://www.cs4fn.org/ai/snap/ } \\
\text { http://www.cs4fn.org/teachers/activities/sweetcomputer/sweetcomputer.pdf }\end{array}$ \\
\hline (Curiositymachine, 2019) & Curiosity Machine & https://www.curiositymachine.org/challenges/126/ \\
\hline (Elements of AI, 2019) & Elements of AI & https://course.elementsofai.com/4 \\
\hline (Essinger \& Rosen, 2019) & $\begin{array}{l}\text { Machine Learning: An } \\
\text { Introductory Unit of Study for } \\
\text { Secondary Education }\end{array}$ & $\begin{array}{l}\text { Essinger, S. D., Rosen, G. L.(2019) Machine Learning: An Introductory Unit of Study for } \\
\text { Secondary Education. Proc. of the 50th ACM Technical Symposium on Computer Science } \\
\text { Education, Minneapolis, MN, USA. }\end{array}$ \\
\hline (Estevez et al., 2019) & $\begin{array}{l}\text { Gentle Introduction to Artificial } \\
\text { Intelligence for High-School } \\
\text { Students Using Scratch }\end{array}$ & $\begin{array}{l}\text { Estevez, J. et al. "Gentle Introduction to Artificial Intelligence for High-School Students } \\
\text { Using Scratch," in IEEE Access, vol. 7, pp. 179027-179036, 2019, doi: } \\
\text { 10.1109/ACCESS.2019.2956136. }\end{array}$ \\
\hline (Evangelista et al., 2018) & $\begin{array}{l}\text { Why are we not teaching machine } \\
\text { learning at high school? }\end{array}$ & $\begin{array}{l}\text { Evangelista, I. et al. (2018). Why Are We Not Teaching Machine Learning at High School? } \\
\text { A Proposal. Proc. of the World Engineering Education Forum - Global Engineering Deans } \\
\text { Council, Albuquerque, NM, USA. }\end{array}$ \\
\hline $\begin{array}{l}\text { (Exploring Computer } \\
\text { Science, 2019) }\end{array}$ & $\begin{array}{l}\text { Alternate Curriculum Unit: } \\
\text { Artificial Intelligence }\end{array}$ & http://www.exploringcs.org/for-teachers-districts/artificial-intelligence \\
\hline (Fryden curriculum, 2019) & Fryden Curriculum & http://www.fryden-learning.com/fryden-curriculum \\
\hline (García et al., 2019) & $\begin{array}{l}\text { Developing Computational } \\
\text { Thinking at School with Machine } \\
\text { Learning: An exploration }\end{array}$ & $\begin{array}{l}\text { J. D. R. García, et al. (2019) Developing Computational Thinking at School with Machine } \\
\text { Learning: An exploration. Proc. of the Int. Symposium on Computers in Education, Tomar, } \\
\text { Portugal, 1-6. }\end{array}$ \\
\hline
\end{tabular}




\begin{tabular}{|c|c|c|}
\hline (Hitron et al., 2019) & \begin{tabular}{|l|} 
Can Children Understand \\
Machine Learning Concepts?: The \\
Effect of Uncovering Black \\
Boxes.
\end{tabular} & $\begin{array}{l}\text { Hitron, T. et al. (2019). Can Children Understand Machine Learning Concepts?: The Effect } \\
\text { of Uncovering Black Boxes. Proc. of the CHI Conference on Human Factors in Computing } \\
\text { Systems, Glasgow, Scotland UK. }\end{array}$ \\
\hline (Ho \& Scadding, 2019) & $\begin{array}{l}\text { Classroom Activities for Teaching } \\
\text { Artificial Intelligence to Primary } \\
\text { School Students }\end{array}$ & $\begin{array}{l}\text { Ho,J. W. K., Scadding, M. (2019). Classroom Activities for Teaching Artificial Intelligence } \\
\text { to Primary School Students. Proc. of the Int. Conference on Computational Thinking, Hong } \\
\text { Kong, China. }\end{array}$ \\
\hline $\begin{array}{l}\text { (Kahn \& Winters, 2018) } \\
\text { (Kahn et al., 2018) }\end{array}$ & eCraft2Learn & $\begin{array}{l}\text { Kahn, K., Winters, N. (2018) AI Programming by Children. Proc. of the Constructionism } \\
\text { Conf., Vilnius, Lithuania. Kahn, K. et al. (2018) AI Programming by Children using Snap! } \\
\text { Block Programming in a Developing Country. In Proc. of the European Conf. on } \\
\text { Technology Enhanced Learning, Delft, Netherlands. }\end{array}$ \\
\hline $\begin{array}{l}\text { (Mariescu-Istodor \& } \\
\text { Jormanainen, 2019) }\end{array}$ & $\begin{array}{l}\text { Machine Learning for High } \\
\text { School Students }\end{array}$ & $\begin{array}{l}\text { Radu Mariescu-Istodor and Ilkka Jormanainen. 2019. Machine Learning for High School } \\
\text { Students. Proc. of the 19th Koli Calling Int. Conf. on Computing Education Research, ACM, } \\
\text { New York, NY, USA, Article 10,1-9. }\end{array}$ \\
\hline (MIT App Inventor, 2019) & $\begin{array}{l}\text { Introduction to Machine Learning: } \\
\text { Image Classification }\end{array}$ & tp://appinventor.mit.edu/explore/resources/ai/image-classification-look-extension \\
\hline (MIT, 2019b) & $\begin{array}{l}\text { How to train your robot } \\
\text { companion }\end{array}$ & https://aieducation.mit.edu/documents/i2educatorguide.pdf \\
\hline (MIT, 2020c) & Creative AI & $\begin{array}{l}\text { https://docs.google.com/document/d/1NzFg9CV25aiPKL0Smn6mBQ_LOWV4ayH7oxbSC } \\
\text { HMJr48/edit\# }\end{array}$ \\
\hline (ML4Kids, 2019) & Machine Learning for Kids & https://machinelearningforkids.co.uk/\#!/worksheets \\
\hline (Mobasher et al., 2019) & $\begin{array}{l}\text { Data Science Summer Academy } \\
\text { for Chicago Public School } \\
\text { Students }\end{array}$ & $\begin{array}{l}\text { Mobasher, B. et al. (2019). Data Science Summer Academy for Chicago Public School } \\
\text { Students. ACM SIGKDD Explorations Newsletter, 21(1). }\end{array}$ \\
\hline $\begin{array}{l}\text { (Narahara \& Kobayashi., } \\
\text { 2018) }\end{array}$ & $\begin{array}{l}\text { Personalizing homemade bots } \\
\text { with plug and play AI for STEAM } \\
\text { education }\end{array}$ & $\begin{array}{l}\text { Narahara, T., Kobayashi, Y. (2018). Personalizing homemade bots with plug and play AI for } \\
\text { STEAM education. Proc. of SIGGRAPH Asia Technical Briefs, Tokyo, Japan. }\end{array}$ \\
\hline (ReadyAI, 2019) & Ready $\mathrm{AI} \mathrm{AI}+\mathrm{Me}$ & https://edu.readyai.org/courses/aime/ \\
\hline (Sabuncuoglu, 2020) & $\begin{array}{l}\text { Designing One Year Curriculum } \\
\text { to Teach Artificial Intelligence for } \\
\text { Middle School }\end{array}$ & $\begin{array}{l}\text { Sabuncuoglu, A. (2020). Designing one year curriculum to teach Artificial Intelligence for } \\
\text { Middle School. Proc. of the Conf. on Innovation and Technology in Computer Science } \\
\text { Education. ACM, New York, NY, USA, 96-102. }\end{array}$ \\
\hline $\begin{array}{l}\text { (Sakulkueakulsuk et al., } \\
\text { 2018) }\end{array}$ & $\begin{array}{l}\text { Kids making AI: Integrating } \\
\text { machine Learning, gamification, } \\
\text { and social Context in STEM } \\
\text { Education }\end{array}$ & $\begin{array}{l}\text { Sakulkueakulsuk, B. et al. (2018). Kids making AI: Integrating Machine Learning, } \\
\text { Gamification, and Social Context in STEM Education. Proc. of IEEE Int. Conf.on Teaching, } \\
\text { Assessment, and Learning for Engineering, Wollongong, Australia. }\end{array}$ \\
\hline $\begin{array}{l}\text { (Sperling \& Lickerman, } \\
\text { 2012) }\end{array}$ & $\begin{array}{l}\text { Integrating AI and machine } \\
\text { learning in software engineering } \\
\text { course for high school students }\end{array}$ & $\begin{array}{l}\text { Sperling, A., Lickerman, D. (2012). Integrating AI and machine learning in software } \\
\text { engineering course for high school students. Proc. of the 17th ACM Annual Conf. on } \\
\text { Innovation and Technology in Computer Science Education, Haifa, Israel. }\end{array}$ \\
\hline (Srikant \& Aggarwal, 2017) & $\begin{array}{l}\text { Introducing Data Science to } \\
\text { School Kids }\end{array}$ & $\begin{array}{l}\text { Srikant, S., Aggarwal, V. (2017). Introducing Data Science to School Kids. Proc. of the } \\
\text { ACM 48th SIGCSE Technical Symposium on Computer Science Education, Seattle, WA, } \\
\text { USA. }\end{array}$ \\
\hline $\begin{array}{l}\text { (Tang, 2019)(Tang et al., } \\
\text { 2019) }\end{array}$ & $\begin{array}{l}\text { Empowering novices to } \\
\text { understand and use Machine } \\
\text { Learning with personalized image } \\
\text { classification models, intuitive } \\
\text { analysis tools, and MIT App } \\
\text { Inventor. }\end{array}$ & $\begin{array}{l}\text { Tang, D. (2019). Empowering Novices to Understand and Use Machine Learning With } \\
\text { Personalized Image Classification Models, Intuitive Analysis Tools, and MIT App Inventor, } \\
\text { Thesis, MIT, Cambridge, MA, USA. Tang, D., Utsumi, Y., Lao, N. (2019). PIC: A Personal } \\
\text { Image Classification Webtool for High School Students. Proc. of the IJCAI EduAI } \\
\text { Workshop, Sicily, Italy. }\end{array}$ \\
\hline (TechGirlz, 2018) & $\begin{array}{l}\text { Artificial Intelligence: How } \\
\text { Computers Learn }\end{array}$ & tps://www.techgirlz.org/topic/artificial-intelligence-computers-learn/ \\
\hline (Vachovsky et al., 2016) & $\begin{array}{l}\text { Toward More Gender Diversity in } \\
\text { CS through an Artificial } \\
\text { Intelligence Summer Program for } \\
\text { High School Girls }\end{array}$ & $\begin{array}{l}\text { Vachovsky, M. E. et al. (2016). Toward More Gender Diversity in CS through an Artificial } \\
\text { Intelligence Summer Program for High School Girls. Proc. of the 47th ACM Technical } \\
\text { Symposium on Computing Science Education, Memphis, TN, USA. }\end{array}$ \\
\hline $\begin{array}{l}\text { (Van Brummelen, 2019) } \\
\text { (Van Brummelen \& Abelson, } \\
\text { 2018) (Van Brummelen et } \\
\text { al., 2019) }\end{array}$ & $\begin{array}{l}\text { App Inventor for Conversational } \\
\text { AI }\end{array}$ & $\begin{array}{l}\text { Van Brummelen, J. (2019). Tools to Create and Democratize Conversational Artificial } \\
\text { Intelligence. Thesis, MIT,, Cambridge MA, USA._Van Brummelen, J., Abelson, H. (2018). } \\
\text { What's conversational AI?' with MIT App Inventor and Amazon Alexa. Proc. of Amazon } \\
\text { Research Days, Boston, MA, USA. Van Brummelen, J., Shen, J. H., Patton, E. W. (2019).. } \\
\text { The Popstar, the Poet, and the Grinch: Relating Artificial Intelligence to the Computational } \\
\text { Thinking Framework with Block-based Coding. Proc. of the Int. Conf. on Computational } \\
\text { Thinking, Hong Kong, China. }\end{array}$ \\
\hline $\begin{array}{l}\text { (Wong et al. 2019)(Wong et } \\
\text { al. 2020) }\end{array}$ & $\begin{array}{l}\text { When schools meet artificial } \\
\text { intelligence in Hong Kong }\end{array}$ & $\begin{array}{l}\text { Wong, G. et al. 2019. When schools meet artificial intelligence in Hong Kong. ACM } \\
\text { Inroads 10(4), 43-46. }\end{array}$ \\
\hline (Zhu, 2019) & $\begin{array}{l}\text { An Educational Approach to } \\
\text { Machine Learning with Mobile } \\
\text { Applications }\end{array}$ & $\begin{array}{l}\text { Zhu, K. (2019). An Educational Approach to Machine Learning with Mobile Applications. } \\
\text { Thesis, MIT, Cambridge, MA, USA. }\end{array}$ \\
\hline $\begin{array}{l}\text { (Zimmermann- Niefield et } \\
\text { al., 2019a)(Zimmermann- } \\
\text { Niefield et al., 2019b) }\end{array}$ & $\begin{array}{l}\text { Sports and machine learning: how } \\
\text { young people can use data from } \\
\text { their own bodies to learn about } \\
\text { machine learning }\end{array}$ & $\begin{array}{l}\text { Zimmermann-Niefield, A. et al. (2019a). Sports and machine learning: How young people } \\
\text { can use data from their own bodies to learn about machine learning. XRDS: Crossroads, } \\
\text { 25(4), 44-49. Zimmermann-Niefield, A. et al. (2019b).. Youth Learning Machine Learning } \\
\text { through Building Models of Athletic Moves. Proc. of the 18th ACM Int. Conf. on Interaction } \\
\text { Design and Children, Boise, ID, USA. }\end{array}$ \\
\hline
\end{tabular}


Isso mostra que até o momento, poucas UIs abordam o ensino de ML na educação básica. A maior parte delas é também muito recente demonstrando a crescente importância de IA/ML nos últimos anos.

\subsection{Quais competências de ML são ensinadas nas UI?}

As UIs ensinam competências variando desde o que é ML, até algumas técnicas específicas. Entre os tópicos mais frequentemente abordados estão IA, redes neurais, e uma introdução referente ao que significa aprender (Figura 1). Muitas UIs também apresentam outros algoritmos de ML, como árvore de decisão e/ou aprendizagem baseada em instâncias, geralmente utilizando atividades unplugged. Poucas UIs abordam o tópico de implicações sociais e questões éticas.

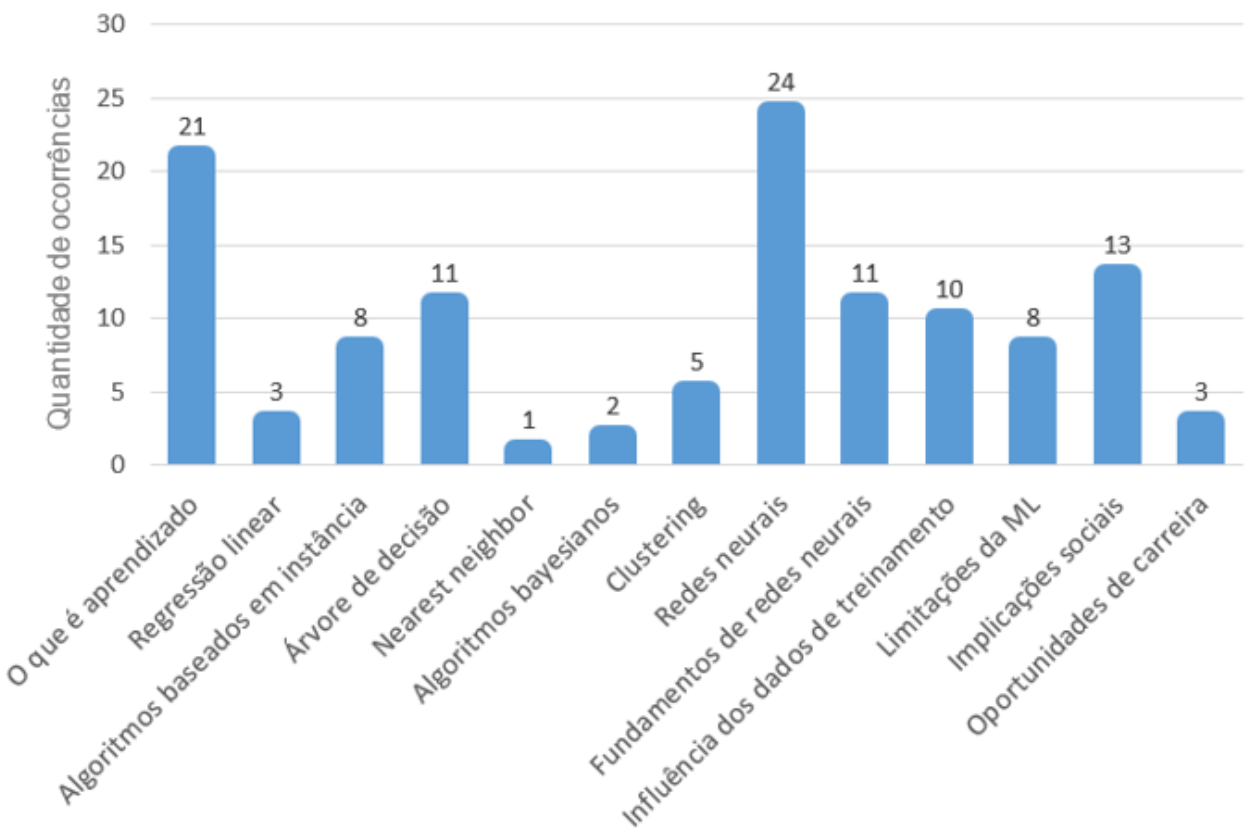

Figura 1. Frequência de abordagem de tópicos de ML

Muitas UIs abordam o tópico de redes neurais, geralmente trazendo esse conteúdo de forma teórica e/ou por meio de aplicações práticas. Entretanto, algumas UIs apenas proporcionam um entendimento genérico dos mecanismos de ML e suas aplicações. A maioria das UIs aborda apenas um algoritmo de ML apresentando um exemplo, demonstração ou atividades práticas.

De forma geral, as UIs se esforçam para demonstrar a aplicação de ML na prática, apresentando vários exemplos para obter a atenção dos estudantes. Isso inclui principalmente a demonstração da classificação em tarefas de visão computacional, como reconhecimento facial ou de gestos [Hitron et al. 2019].

Observando a complexidade dos conceitos de ML, muitas UIs abordam apenas os processos mais acessíveis, como gerenciamento de dados [Mobasher et al. 2019] [Srikant \& Aggarwal 2017]. Por outro lado, um número considerável de UIs também inclui as fases de aprendizado de modelo e teste em níveis diferentes de profundidade. Porém, a maior parte dessas UIs apresenta muitos conceitos apenas num nível abstrato, escondendo alguns processos subjacentes de ML. Nesses casos, o processo de aprendizagem é abordado apenas 
executando um processo pré definido (como em ML4Kids (2019)). Poucas UIs abordam a avaliação do desempenho de ML, geralmente apresentando somente acurácia de uma forma superficial. Apenas um pequeno número de UIs incluem também a fase de implantação (deployment) do modelo de ML criado por meio de aplicações móveis, por exemplo.

Diferentes frameworks e ferramentas de ML são utilizados para minimizar a complexidade da fase de treinamento do modelo de ML (Figura 2). Por exemplo, ML4kids (2019) provê uma interface usada nesse estágio com o objetivo de abstrair alguns conceitos, permitindo que pessoas inexperientes treinem uma rede neural sem uso de programação. Como são tipicamente utilizadas no ensino de computação na educação básica, as UIs de ML também adotam predominantemente linguagens de programação baseadas em blocos, como Scratch, Snap! ou App Inventor.

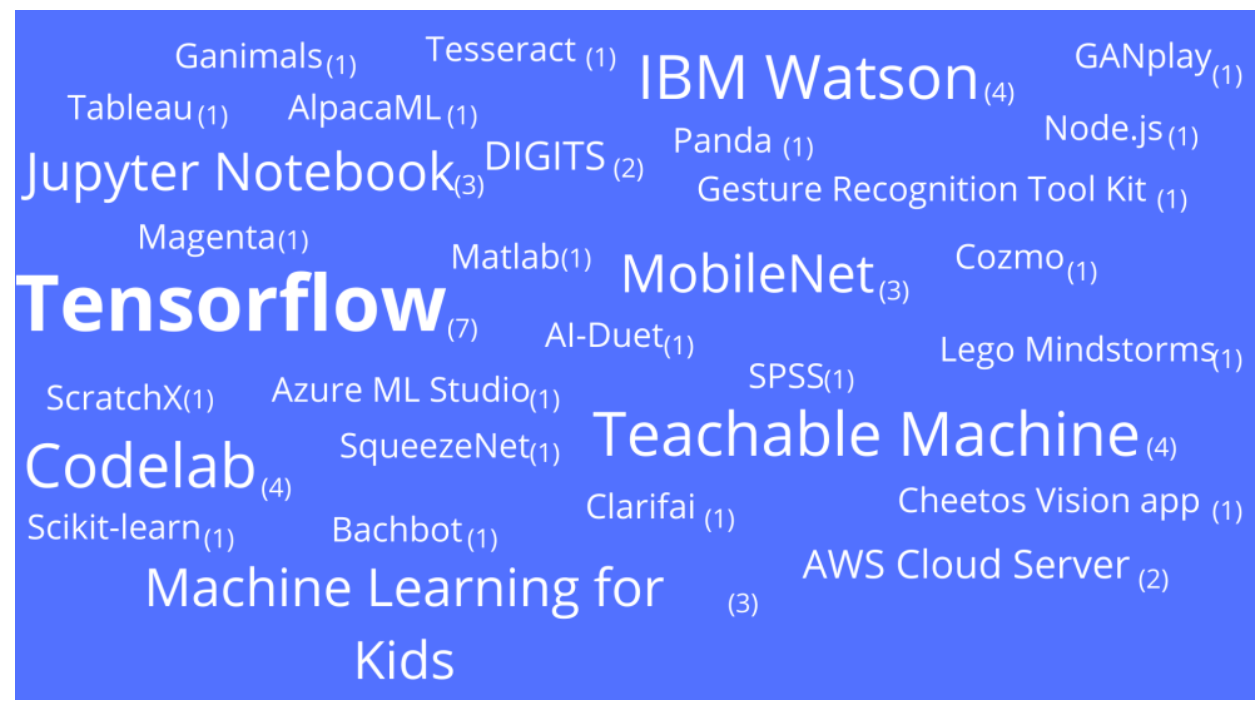

Figura 2. Frequência de frameworks/ferramentas de $M L$ utilizadas

Por outro lado, algumas UIs usam frameworks de ML e/ou ambientes baseados em linguagens de programação textual como TensorFlow e Jupyter Notebooks. Seis UIs usam Python como linguagem de programação.

\subsection{Quais são as características instrucionais das UIs?}

Como o ensino de ML não está tipicamente incluído no ensino de computação, a maioria das UIs são propostas como atividades extracurriculares, com apenas MIT (2019a), Sperling and Lickerman (2012), Exploring Computer Science (2019) e Sabuncuoglu (2020) propondo uma unidade curricular. Três cursos online foram encontrados [ReadyAI 2019][Elements of AI 2019][Kahn \& Winters 2018]. Levando em conta a atual falta de conhecimento relacionado à computação e/ou ML, muitas unidades instrucionais focam em iniciantes sem competências prévias nessas áreas. A maioria das UIs são focadas em ensinar ML no ensino médio. Mesmo assim, existe um número considerável de UIs também para o ensino fundamental, indicando que a inserção de ML pode ser benéfica desde os primeiros níveis escolares.

A duração das IUs varia bastante de atividades curtas (45 min.) até cursos com duração de semanas, embora na maioria sejam curtas. Referente aos métodos instrucionais, se observa uma forte predominância de atividades práticas, que levem o estudante ao entendimento por meio da aplicação dos conceitos. Adicionalmente, algumas UIs utilizam métodos como leitura, vídeo e demonstração, bem como desafios e discussões mediadas. Vachovsky et al. (2016) e Mobasher et al. (2019) também incluem conversas com 
profissionais da área, para ampliar a perspectiva dos estudantes no que se refere à ML como profissão. Muitos tipos de materiais instrucionais são adotados, porém observa-se uma falta de informação em relação à disponibilidades e licenças, bem como poucas opções em línguas além do Inglês.

A maioria das UIs não definem a forma de avaliação da aprendizagem dos alunos. Somente AI Family challenge (2019), AlinSchools (2019), Exploring Computer Science (2019) e MIT (2019b) propõem uma folha de rubrica/avaliação para analisar as criações dos estudantes. Sakulkueakulsuk et al. (2018) propõe um sistema de pontos com base na acurácia do modelo de ML desenvolvido.

\section{Discussão}

Considerando que atualmente o ensino de ML na educação básica é um tema emergente, um número considerável de UIs foi encontrado. Essas IUs, com foco principalmente em iniciantes em qualquer estágio educacional do ensino fundamental ao médio, também indicam o reconhecimento de uma exposição precoce dos alunos aos conceitos de ML, não se limitando apenas ao ensino médio, como normalmente indicado por diretrizes de currículo de computação. Por ser um tema emergente, a maioria das UIs são propostas como unidades extracurriculares tanto de forma presencial quanto como cursos online. Fornecendo diversos materiais didáticos disponíveis gratuitamente, essas UIs facilitam sua aplicação prática. Várias também fornecem ferramentas customizadas para ensinar ML nesta fase educacional usando, por exemplo, ambientes visuais para a criação de modelos de ML sem a necessidade de programação. No entanto, como a maioria das UIs está disponível apenas em inglês, a sua aplicação em outros países é dificultada. Outro problema é a falta de informações sobre a avaliação da aprendizagem dos alunos, o que é importante como feedback para o aluno e instrutor a fim de orientar o processo de aprendizagem.

As UIs ensinam competências que vão desde a apresentação do que é ML, até técnicas específicas de ML, bem como os impactos da ML. No entanto, observamos que várias apresentam conceitos de ML apenas em um nível abstrato, omitindo alguns dos processos de ML subjacentes, mesmo como parte de atividades práticas, a fim de reduzir a complexidade. No entanto, em alguns casos, esse alto nível de "caixa preta" pode limitar os alunos na exploração e construção de modelos mentais de ML. Por outro lado, algumas UIs também abordam o aprendizado de como aplicar conceitos de ML a problemas práticos no que diz respeito às mais diversas tarefas do contexto dos alunos, contribuindo positivamente também na sua motivação.

Ameaças à validade. Mapeamentos sistemáticos podem sofrer da tendência de que resultados positivos são mais prováveis de serem publicados do que negativos. Entretanto, consideramos que para este mapeamento, as abordagens das UIs são mais relevantes do que a análise de seus resultados, assim consideramos esse risco mínimo. Também corremos o risco de ter omitido algum resultado relevante. Para mitigar esse risco, construímos cuidadosamente a string de busca para ser o mais inclusiva possível, considerando não apenas os conceitos principais, mas também seus sinônimos. Além disso, considerando a atualidade dos estudos temáticos, também buscamos por qualquer UI disponível online, não apenas considerando artigos científicos, a fim de reduzir o risco de exclusão de UIs existentes. Ameaças à seleção de UIs relevantes e extração de dados foram mitigadas fornecendo uma definição detalhada de critérios de inclusão/exclusão. Definimos e documentamos um protocolo rígido para a seleção dos estudos e todos os autores realizaram a seleção juntos, discutindo a seleção até 
que um consenso foi alcançado. A extração de dados foi dificultada em alguns casos, pois as informações relevantes nem sempre eram apresentadas explicitamente e, portanto, em alguns casos, tiveram que ser inferidas. No entanto, essa inferência foi feita pelos dois primeiros autores e cuidadosamente revisada pelo terceiro autor.

\section{Conclusão}

Como resultado do mapeamento, identificamos 39 UIs focadas principalmente em iniciantes, seja qual for seu nível educacional. Por ser um tema emergente, a maioria das UIs estão propostas como unidades extracurriculares, variando os conteúdos desde os conceitos básicos até a aplicação deles em atividades práticas usando ferramentas e ambientes que facilitem a construção de modelos de ML. Foi notável a dificuldade em encontrar registros do planejamento e desenvolvimento das UIs, bem como suas avaliações. Desse modo a aplicação destas UIs se torna mais complexa, por possuir muitas vezes poucas informações além de incerteza em relação aos impactos de sua aplicação. A falta de UIs disponíveis de forma mais ampla em diversas línguas como Português, indica a necessidade de pesquisa e ação nessa área para contribuir para a democratização desse conhecimento também em escolas brasileiras.

\section{Agradecimentos}

Este trabalho foi apoiado pelo CNPq (Conselho Nacional de Desenvolvimento Científico e Tecnológico - www.cnpq.br), entidade do governo brasileiro voltada ao desenvolvimento científico e tecnológico.

\section{Referências}

Avila, C., et al. (2017) "O Pensamento Computacional por meio da Robótica no Ensino Básico - Uma Revisão Sistemática”. In Anais do Simpósio Brasileiro de Informática na Educação, Recife, Brasil.

Bordini, A. et al. (2017) "Pensamento Computacional nos Ensinos Fundamental e Médio: uma revisão sistemática". In Anais do Simpósio Brasileiro de Informática na Educação, Recife, Brasil.

Evangelista, I. et al. (2018). Why are we not teaching machine learning at high school? A proposal. In: Proc. of the World Engineering Education Forum - Global Engineering Deans Council, Albuquerque, NM, USA.

Ferreira, M. N. F. et al. (2019) "Ensinando Design de Interface de Usuário na Educação Básica: Um Mapeamento Sistemático do Estado da Arte e Prática”. In Anais do Workshop de Informática na Escola, Brasília, Brasil.

Forbes. (2019) "AI goes to high school". https://www.forbes.com/sites/insightsintelai/2019/05/22/ai-goes-to-high-school/\#68826e3f1d0c

Google. (2016) "Trends in the State of Computer Science in U.S. K-12 Schools". $\mathrm{http}$ //services.google.com/fh/files/misc/trends-in-the-state-of-computer-science-report.pdf

Grover, S., Pea, R. (2013) "Computational Thinking in K-12: A Review of the State of the Field”. Educational Researcher, 42(1), 38-43.

Haddaway, N. R. et al. (2015) "The role of Google Scholar in evidence reviews and its applicability to grey literature searching" PloS one, 10(9). 
Heintz, F. et al. (2016) "A Review of Models for Introducing ComputationalThinking, Computer Science and Computing in K-12 Education". In Proc. of IEEE Frontiers in Education Conference, Erie, PA, USA, 1-9.

Hiner, J. (2017) “AI will eliminate $1.8 \mathrm{M}$ jobs but create $2.3 \mathrm{M}$ by 2020 , claims Gartner". https://www.techrepublic.com/article/ai-will-eliminate-1-8m-jobs-but-create-2-3m-by2020-claims-gartner

Hubwieser, P. et al. (2015) "A Global Snapshot of Computer Science Education in K-12 Schools". In Proc. of the ITiCSE on Working Group Reports, Vilnius, Lithuania.

Kandlhofer, M. et al. (2016) "Artificial Intelligence and Computer Science in Education: From Kindergarten to University". In Proc. of IEEE Frontiers in Education Conference, Erie, PA, USA.

Lye, S. Y., Koh, J. H. L. (2014) "Review on teaching and learning of computational thinking through programming: What is next for K-12?" Computers in Human Behavior, 41, 51-61.

Magno Jesus, A. et al. (2019) "Desenvolvimento do Pensamento Computacional por Meio da Colaboração: uma revisão sistemática da literatura". Revista Brasileira de Informática na Educação, 27(2).

McGovern, A. et al. (2011) "Teaching Introductory Artificial Intelligence through JavaBased Games". In Proc. of the 2nd Symposium on Educational Advances in Artificial Intelligence, San Francisco, CA, USA.

Petersen, K. et al. (2008) "Systematic mapping studies in software engineering". In Proc. of the 12th International Conference on Evaluation and Assessment in Software Engineering, Bari, Italy, 68-77.

Royal Society. (2017) "Machine learning: the power and promise of computers that learn by example" https://royalsociety.org/ /media/policy/projects/machinelearning/publications/machine-learning-report.pdf

Tavares, L. A. et al. (2020) "Inteligência Artificial na Educação: Survey”. Brazilian Journal of Development, 6(7).

Torrey, L. (2012) “Teaching Problem-Solving in Algorithms and AI". In Proc. of the 3rd Symposium on Educational Advances in Artificial Intelligence, Toronto, Canada.

Touretzky, D. S. et al. (2019a) "K-12 Guidelines for Artificial Intelligence: What Students Should Know". In Proc. of the ISTE Conference, Philadelphia, PA, USA.

Touretzky, D. S. et al. (2019b) "Envisioning AI for K-12: What Should Every Child Know about AI?". In Proc. of the $33^{\text {rd }}$ AAAI Conference on Artificial Intelligence, Honolulu, Hawaii, USA.

Touretzky, D. S., et al. (2019c) "K-12 AI Playground". In Proc. of the CSTA Annual Conference, Philadelphia, PA, USA.

Wollowski, M. et al. (2016) "A Survey of Current Practice and Teaching of AI". Proc. of the 30th AAAI Conference on Artificial Intelligence, Phoenix, AZ, USA. 